

ABSTRACT. We present here new results on the periodic modulations in the optical light curves of Ex Hydrae observed during 1982-84. The period analysis has been done using synchronous summation and discrete Eourier transform methods. We report new periodicities at $34.7 \mathrm{~min}$ and $26.7 \mathrm{~min}$ apart from confirming the detection of 46.3 min modulation recently reported. We also include our data to derive the rate of decrease of the $67 \mathrm{~min}$ period.

\title{
1.0. INTRODUCTION
}

EX Hydrae has long been known as an eclipsing binary (Mumford, 1964) having short eclipses recurring with 98.3 min periodicity. Classified recently as an intermediate polar (Warner, 1982), it is a binary system consisting of a white dwarf accreting matter from a red dwarf.

Other periodicities discovered in the system are at 67 minute confirmed to be the rotational period of the white dwarf (Kruszewski et al., 1981, Cordova et al., 1985), and at 46.3 minute (Cordova et al., 1985) at present speculated to be a beat of $1 / 2$ porb (= $49 \mathrm{~min})$ and a longer as yet undiscovered 0.58 day precessional period of the disc.

Paper presented at the IAU Colloquium No. 93 on 'Cataclysmic Variables. Recent Multi-Frequency Observations and Theoretical Developments', held at Dr. Remeis-Sternwarte Bamberg, F.R.G., 16-19 June, 1986. 


\subsection{OBSERVATIONS}

We conducted photometric observations using the 1 metre reflector at the Vainu Bappu Observatory at Kavalur. A dry ice/thermoelectrically cooled RCA 4832/EMI $9804 \mathrm{QB}$ tube was used at the Cassegrain focus of this reflector.

Observations were conducted in March 1982, Jan-Feb 1983 and April 1984. Most of the long runs were taken without filter. Typical integration times used were $1 \mathrm{~s}$ or $2 \mathrm{~s}$.

\subsection{RESULTS AND DISCUSSIONS}

\subsection{Periedicities in the System}

The longest stretch (about $5 \mathrm{hr}$ ) of almost continuous data was obtained on $23 \mathrm{Feb}, 1983$ and this data train was used for the time series analysis. The data was subjected to an epoch folding algorithm (Warner, 1972) and a discrete Fourier transform (Deeming, 1975). The discrete Fourier transform output shown in Figure 1 indicates periods at $67 \mathrm{~min}, 46.3 \mathrm{~min}, 34.7 \mathrm{~min}$, and $26.7 \mathrm{~min}$. These periodicities are also confirmed by the epoch folding algorithm. The 67 min period with a $21 \%$ modulation is the well known rotational period of the white dwarf.

The $46.3 \mathrm{~min}$ period which has a $5 \%$ differential amplitude has been reported earlier only by Cordova et al., 1985. Their observations conducted in March 1983 also exhibit a similar modulation and therefore this periodicity has remained coherent for at least a month.

We report new periods at 34.7 and $26.7 \mathrm{~min}$, with modulations of about $5 \%$ and $4 \%$ respectively. The $34.7 \mathrm{~min}$ period could be a beat, in prograde sense, of the second harmonic of the $67 \mathrm{~min}$ period and a 0.58 day precessional period of the disc. The $26.7 \mathrm{~min}$ period could be the beat of the $46.3 \mathrm{~min}$ and $67 \mathrm{~min}$ periods.

Confirmation of the 0.58 day precessional period of the disc and exposition of models for the system would require additional long term/global monitoring of this system in $\mathrm{X}$-ray and optical bands. 


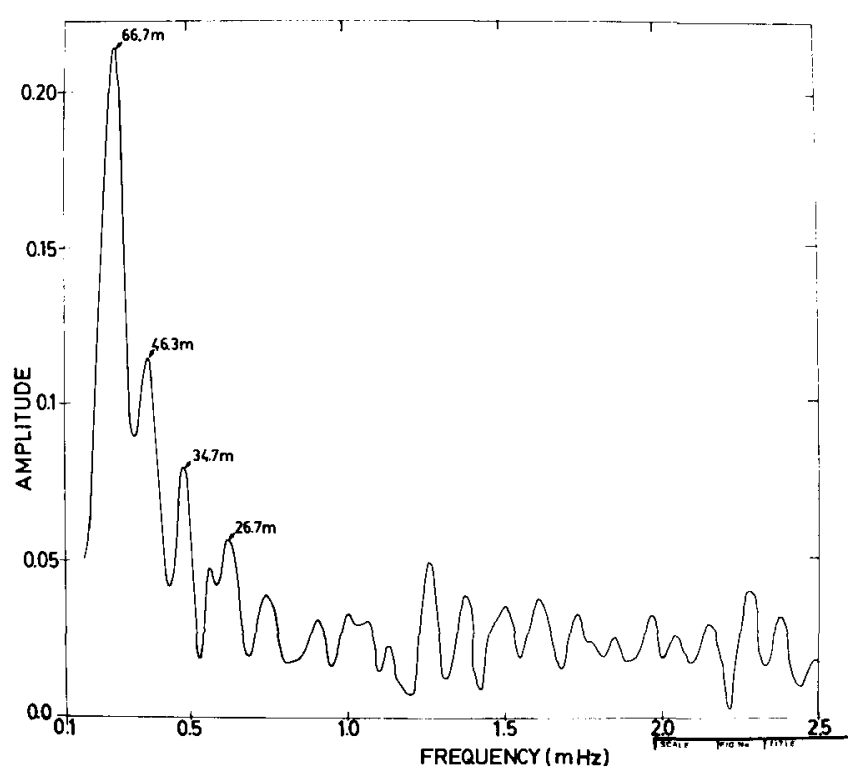

Figure 1. Output of discrete fourier transform.

\section{$3.2 \cdot(0-c)$ calculations}

The decreasing trend of the 67 minute variation has been noticed and reported since 1980 (see Vogt et al., 1980, Kruszewski et al., 1981, Gilliland 1982, sterken et al., 1983). We added the times of maxima (in HJD) of our observations to earlier data set. Using Vogt's linear ephemeris we calculated the $\mathrm{O}-\mathrm{C}$ and $\mathrm{E}$ for the complete data and these values were fitted with a quadratic to yield the following ephemeris :

$$
\begin{aligned}
T_{\max }(H J D)=2437699.8899+0.046546534 \mathrm{E} & -0.9810^{-12} \mathrm{E}^{2} \\
\pm 6 & \pm 18 \pm 0.011
\end{aligned}
$$

Figure 2 shows the plot of $\mathrm{O}-\mathrm{C}$ versus $\mathrm{E}$ for the complete data with the quadratic fit. The rate of decrease of the 67 minute period as derived from the ephemeris given in Equation (I) is

$(4.2 \pm 0.47) 10^{-11} \mathrm{~s} \mathrm{~s}^{-1}$. This gives $\mathrm{P} / \dot{\mathrm{P}}=3 \times 10^{6}$ years. 




Figure 2. (O-C) versus cycle number.

\section{ACKNOWLEDGEMENTS}

The authors profusely thank Prof. R. Staubert for his invaluable help, to develop the epoch folding algorithm, Mr. P. Padmanaban for useful discussions and suggestions, and Mrs. Padmavathy for her secretarial assistance for the preparation of this paper.

\section{REFERENCES}

Cordova, F.A. et al., MNRAS, 1985, 212, 447.

Deeming, T.J., Astrophys Space Science, 1975, 36, 137.

Gilliland, R.L., Ap.J,, 1982, 258, 576.

Kruszewski, A. et al., Sp.Sci.Rev,, 1981, 30, 221.

Murford, G.S., PASP, 1964, 76, 57.

Sterken, C. et al., Astron and astrophys, 1983, 118, 325.

Vogt, N. et al., Astron and astrophys, 1980, 85, 106.

Warner, B. and Robinson, E.L., MNRAS, 1972, 159, 101.

Warner, B. and Mc Graw, J.T., MNRAS, 1981, 196, 59.

Warner, B., 'Cataclysmic Variables and Related Objects', edited by Mario Livio and Giora Shaviv, P155, D. Reidel Publ. Co. 1982 . 